\title{
Enhancement of Raman scattering from molecules placed near metal nanoparticles
}

\author{
B. Barbiellini \\ Department of Physics, Northeastern University, Boston, MA 02115, USA \\ E-mail: B.Amidei@neu.edu
}

Received August 1, 2016, published online November 25, 2016

\begin{abstract}
Large Raman scattering cross sections from molecules on surfaces of metallic nanoparticles are described within a renormalization-group theory. In this approach the valence electrons of the molecules are embedded in an effective medium described by a dielectric function, which integrates out the effect of the plasmonic excitations of the metallic nanoparticles. The source of the enhanced photon inelastic scattering is produced by the resonant excitation of surface plasmons at the metallic nanoparticles. A similar theory has been successfully used to explain the resonant $\mathrm{x}$-ray inelastic scattering and the behavior of nonlinear susceptibilities at the x-ray edges.
\end{abstract}

PACS: 78.30.-j Infrared and Raman spectra;

78.67.Bf Nanocrystals, nanoparticles, and nanoclusters.

Keywords: SERS, surface Plasmon, renormalization-group theory.

\section{Introduction}

Einstein's hypothesis of granular light [1], previously based on the photoelectric effect [2], was confirmed by the discovery of the Compton effect. The experiment [3] detected a modification in wavelength of an x-ray quantum when scattering with a free electron in a material. The resulting change in energy and momentum showed that the photon behaves like a particle colliding with the electron [4]. Later on, Raman and Krishnam [5] observed a similar effect in liquids, which involves a change in wavelength of monochromatic visible light scattering with phonons. The effect was also observed independently in crystal by Landsberg and Mandelstam [6]. Thus, the so-called Raman effect further confirmed the quantum theory of light. Subsequently, Raman scattering became a standard spectroscopy used to characterize molecular and material structures [7]. Nowadays, Raman scattering is also deployed to study excitations in correlated systems such as high temperature oxide superconductors [8].

In general the inelastic scattering cross section of photons is very small [9] and can be cast as the product of the Thomson scattering differential cross section $d \sigma_{\mathrm{Th}} / d \Omega$ with the dynamical structure factor $S(\mathbf{q}, \omega)$ :

$$
\frac{d^{2} \sigma}{d \Omega d \omega}=\frac{d \sigma_{\mathrm{Th}}}{d \Omega} S(\mathbf{q}, \omega) .
$$

The Thomson scattering cross section is determined by the classical electron radius $r_{0}$

$$
\sigma_{\mathrm{Th}}=\frac{8 \pi}{3} r_{0}^{2}
$$

This radius occurs in theory of phenomena considered as classical $[10,11]$ and it is given by

$$
r_{0}=\frac{e^{2}}{4 \pi \varepsilon_{0} m c^{2}}=2.82 \cdot 10^{-13} \mathrm{~cm},
$$

where $e$ is the electron charge, $m$ is the electron mass, $c$ is the speed of light and $\varepsilon_{0}$ is the vacuum dielectric constant. The dynamical structure factor $S(\mathbf{q}, \omega)$ describes various excited states involving phonons or particle-hole excitations, which produce a change in momentum $q$ and a change in energy $\omega$ of the scattered photons.

The surface-enhanced Raman scattering (SERS), involving molecules near metallic nanoparticles [12,13], was first observed in 1974 by Fleischmann et al. [14]. After this discovery, further experiments $[15,16]$ explained SERS as a giant enhancement of the ordinary Raman scattering. The enhancement of Raman scattering signals from molecules absorbed on nanostructured metal surfaces can reach 6 to 10 orders of magnitude [15,17]. Moreover, an enhancement about 14 orders of magnitude was observed on molecules near silver aggregates [18]. Therefore, this striking effect can enable single molecule detection. The 
enhancement mechanism in SERS seems to be well understood within the classical plasmonic theory [19-23]. This theory claims that SERS arises from the concentration of electromagnetic energy in the vicinity of an a metal nanoparticle, when the incident light excites surface plasmons. Semiclassical theories involving many-electron Green's function techniques have also been considered [24]. Interestingly, models combining Quantum Mechanics and Maxwell's equation are presently used to describe hybrid systems composed of molecules (or quantum dots) and metallic nanoparticles [25]. Unfortunately, all these models neglect the granular behavior of light.

The present paper explores the possibility to use a renormalization-group approach [26] to derive the fundamental origin of the enhancement mechanism of SERS. This method is beyond perturbation theory and can take in account collision processes between light quanta and the elementary excitations of molecules at metallic surfaces. A similar approach has also been used to simplify the description of the resonant $x$-ray inelastic scattering (RIXS) for the particle-hole excitations [9]. Moreover, within the same scheme, one can derive an expression for the resonant term of the nonlinear susceptibility at x-ray edges [27].

\section{Theory}

The dimensionless coupling of quantum electrodynamics (QED) is not constant but varies under changes of scales [28-30]. In practice, the QED vacuum can be considered as a polarizable system, where virtual pairs of particles and anti-particles screen the electric charge. For instance, the dimensionless coupling changes at the crossing of particle production thresholds in the standard model of particle physics [31]. Similarly, in materials such as FeTe or $\mathrm{TiSe}_{2}$ [9], photons with energies near the $L_{3}$ absorption threshold produce virtual intermediate states involving a $2 p$ core hole and electron excited in a $3 d$ state. In the case of SERS, the threshold energy is given by the plasmonic resonance of the metal nanoparticles. All these resonant effects can be captured by an effective dielectric function for the valence electrons of the system (here, the molecule). Consequently, the dimensionless coupling $g$ in the system can decrease by subjecting the system to a flux photons having energies just below resonant edges, thereby increasing the dielectric screening of the valence electrons of the system. Thus, the inelastic scattering cross section $\sigma$ is enhanced because of an increased background polarizability. The link between the effective coupling $g$ and the length scale $\ell=\sqrt{\sigma}$ is given by

$$
\beta(g)=\frac{d g}{d \ln (\ell)},
$$

where the function $\beta(g)=-3 g^{2}$ is a renormalization group function [26] determining the scale $\ell$ for a given cou- pling $g$. By solving Eq. (4), one finds that the inelastic scattering cross section $\sigma$ is renormalized by

$$
\eta=\exp \left[\frac{2}{3 \alpha}\left(\varepsilon_{1} / \varepsilon_{0}-1\right)\right]
$$

where $\alpha$ is the fine structure constant (i.e., the effective coupling $g$ when $\varepsilon=\varepsilon_{0}$ ) and $\varepsilon_{1}$ is the real part of an effective dielectric function $\varepsilon$. Near the plasmonic absorption edges, there are anomalous dispersions that allow the inelastic scattering cross section to be enhanced in the domain where $\varepsilon_{1} / \varepsilon_{0}$ is larger than unity.

\section{Discussion and conclusion}

We now consider a Raman scattering molecule placed near a metallic nanoparticle. Both the molecule and the metallic particle are subjected to a monochromatic light field. When the incident photon energy is tuned near to the plasma resonance, the effective coupling constant for the valence electron of the molecule is modified through the effective dielectric response, which integrates out the effect of the plasmonic excitations at the metallic nanoparticles. As explained before, the cross section depends strongly on the effective coupling constant, which is inversely proportional to $\varepsilon_{1}$. Figure 1 shows the evolution of the renormalization factor as function of $\varepsilon_{1} / \varepsilon_{0}$. Interestingly, an increase of $\varepsilon_{1} / \varepsilon_{0}$ as small as $30 \%$ can explain enhancements of 14 orders of magnitude. Figure 1 also illustrates that $\varepsilon_{1} / \varepsilon_{0}>1$ is a crucial condition for the resonant enhancement. In fact, when this ratio is smaller than one, the Raman signal weakens instead of growing.

In the RIXS theory, $\varepsilon_{1}$ can be evaluated by using firstprinciples calculations [9]. The model is then able to capture the overall amplitude of the RIXS signal. The reason for small discrepancies between model and experiment above the absorption thresholds can be explained by the fact that the model does not include the momentum inde-

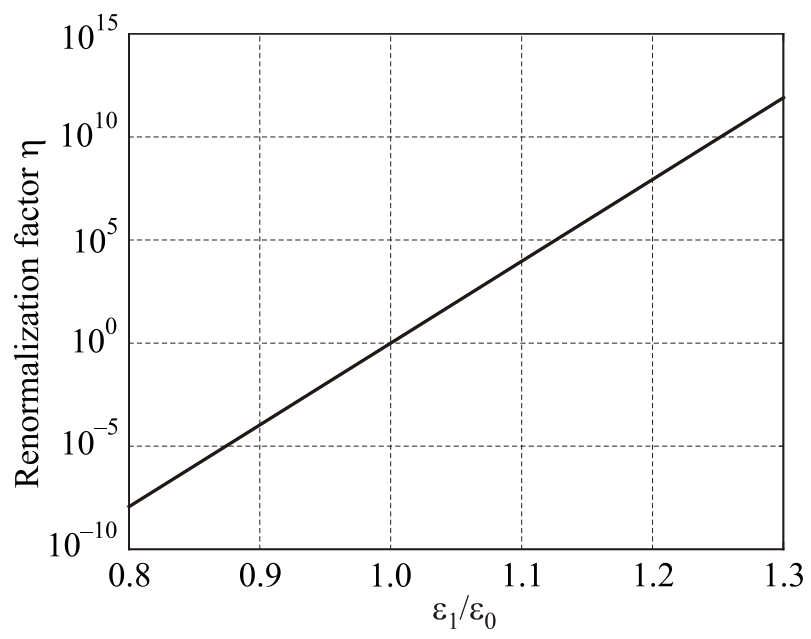

Fig. 1. Cross section enhancement $\eta$ as a function of the ratio $\varepsilon_{1} / \varepsilon_{0}$. 
pendent resonant emission described by the KramersHeisenberg theory [32].

For SERS the $a b$ initio determination of $\varepsilon_{1}$ is still lacking. Therefore, the connection with first-principles calculations must be developed to validate the present theory and to guide future experiments in this field. Clearly, surface plasmon resonances can be exploited to enhance the Raman cross section of molecules placed in vicinity of the metallic nanoparticles. The renormalization group method presented here could offer a powerful method in order to predict huge enhancement factors.

This work is supported by the US Department of Energy (DOE), Office of Science, Basic Energy Sciences grant number DE-FG02-07ER46352 (core research), and benefited from Northeastern University's Advanced Scientific Computation Center (ASCC), the NERSC supercomputing center through DOE grant number DE-AC02-05CH11231, and support (applications to layered materials) from the DOE EFRC: Center for the Computational Design of Functional Layered Materials (CCDM) under DE-SC0012575.

1. A. Einstein, Ann. Phys. 17, 132 (1905).

2. P. Lenard, Ann. Phys. 8, 132 (1902).

3. A.H. Compton, Phys. Rev. 21, 483 (1923).

4. M.I. Kaganov and I.M. Lifshitz, Quasiparticles, MIR Publishers, Moskow (1979).

5. C.V. Raman and K.S. Krishnan, Nature 121, 501 (1928).

6. G. Landsberg and L. Mandelstam, Naturwissenschaften 16, 557 (1928).

7. G. Glocker, Rev. Mod. Phys. 15, 111 (1943).

8. T.P. Devereaux and R. Hackl, Rev. Mod. Phys. 79, 175 (2007).

9. B. Barbiellini, J.N. Hancock, C. Monney, Y. Joly, G. Ghiringhelli, L. Braicovich, and T. Schmitt, Phys. Rev. B 89, 235138 (2014).

10. M. Born, Nature 168, 146 (1952).

11. M.A. Melvin, Nature 171, 890 (1953).

12. M. Moskovits, Rev. Mod. Phys. 57, 783 (1985).
13. B. Sharma, R.R. Frontiera, A.-I. Henry, E. Ringe, and R.P. Van Duyne, Materialstoday 1516 (2012).

14. M. Fleischmann, P.J. Hendra, and A.J. McQuillan, Chem. Phys. Lett. 26, 163 (1974).

15. D.L. Jeanmaire and R.P. Van Duyne, J. Electroanal. Chem. 84, 1 (1977).

16. M.G. Albrecht and J.A. Creighton, J. Am. Chem. Soc. 99, 5215 (1977).

17. K. Kneipp and D. Fassler, Chem. Phys. Lett. 106, 498 (1984).

18. K. Kneipp, Y. Wang, H. Kneipp, L.T. Perelman, I. Itzkan, R. Dasari, and M.S. Feld, Phys. Rev. Lett. 78, 1667 (1997).

19. S.L. McCall, P.M. Platzman, and P.A. Wolff, Phys. Lett. A 77, 381 (1980).

20. D.S. Wang, M. Kerker, and H.W. Chew, Appl. Opt. 19, 2315 (1980).

21. J.I. Gersten and A. Nitzan, J. Chem. Phys. 75, 1139 (1981).

22. F.J. Garcia-Vidal and J.B. Pendry, Phys. Rev. Lett. 77, 1163 (1996).

23. M. Moskovits, Phys. Chem. Chem. Phys. 15, 5301 (2013).

24. D.J. Masiello, Int. J. Quantum Chem. 114, 1413P1420 (2014).

25. L. Hayati, C. Lane, B. Barbiellini, A. Bansil, and H. Mosallaei, Phys. Rev. B 93, 245411 (2016).

26. B. Barbiellini and P. Nicolini, Phys. Rev. A 84, 022509 (2011).

27. B. Barbiellini, Y. Joly, and Kenji Tamasaku, Phys. Rev. B 92, 155119 (2015).

28. L. Landau, in: Niels Bohr and the Development of Physics, W. Pauli (ed.), McGraw-Hill, New York, (1955), p. 52.

29. N.N. Bogoliubov and D.V. Shirkov, Quantum Fields, Benjamin/Cummings, Reading, MA (1983).

30. G.E. Volovik, The Universe in a Helium Droplet, Oxford University Press, New York (2008).

31. F. Jegerlehner, Nucl. Phys. B 181-182, 135 (2008).

32. H. Agren and F. Gel'mukhanov, J. Electron Spectrosc. Relat. Phenom. 110-111, 153 (2000). 